\title{
A Journal on Cervical Cancer Prediction Using Artificial Neural Networks
}

\author{
K.Deepa , and R. Dharani ${ }^{b}$ \\ a,b \\ M.Kumarasamy College of Engineering, Thalavapalayam, Karur-639 113
}

Article History: Received: 11 January 2021; Accepted: 27 February 2021; Published online: 5 April 2021

\begin{abstract}
Artificial neural networks (ANN) assume a significant part in numerous clinical imaging applications. Cervical cancer ranks the 4th dangerous women cancers in less developed countries due to insufficient trained staffs and medical procedures. The location of cervical malignancy cells utilizes ANN for characterizing the typical and unusual cells in the cervix wall of the uterus. Cervical malignancy location is exceptionally difficult on the grounds that this disease happens with no manifestations. The order between the typical,unusual and malignant cells produces exact outcomes than other manual screening techniques. The ANN utilizes a few models for a simple and precise identification of cervical cells. The main aim of artificial neural networks is to supply right information at a right time. Hence we implement artificial neural techniques with collected data Analysis,to improve the life of an individual and to decrease the death rate of the society respectively.
\end{abstract}

Keywords: Cervical cancer; Dataset collected; Cervix detection; artificial neural techniques.

\section{Introduction}

Cervical tumor ranks the 4th most deadly disease among the world with 5,28,000 cases yearly mainly in least developed countries. These cancer disease occurs due to insufficient trained staffs and examining procedures.This cancer is a very challenging cancer among all the cancers because this occurs without any symptoms. It is the third most dangerous cancers among womens. This disease can be easily curative when it is diagnosed at its initial step, and it can be identified by the physicians only through the diagnostics tests. Treatments will be given according to the stages identified correctly. There are about four stages which is proposed by IFOG(International Federation of Gynecology and Obstetrics).Stages of the cancer cells can be identified by the spreads of the tumors (or) other parts of the affected area[2]. The basic symptoms of this cervical cancer includes, abnormal bleeding after menopause,unusual vaginal discharge, tiredness and weight loss, pain in the pelvis and abdomen and irritation while urinating. The main cause for this cervical cancer also includes the long term infections caused by human papillomavirus(HPV) infections. Cancer cells and tumor cells are developed due to the presence of abnormal cells in the wall of the cervix region.

When the researchers focused on this problem they identified that cervical cancer occurs for the those women who smoke regularly because they have a weak immune system and for those who are infected with HIV and also for those who have undergone some organ transplant, these peoples are infected with the higher rate of disease womens were aged between 30 and 69[3].Gynecologists easily identify swelling around the womb by physical examination (or) pap smear tests. Depending upon the severity of the tissues other methods such as ultrasound, MRI scans, $\mathrm{x}$ ray and CT scans (computed tomography) are used to identify the cancer cells in the deeper areas.

Once the cancer cells are identified treatment will be given based on the stages, the following table represents the main stages[21], and the stages are sub divided into STG IA,STG IB,

STG IA1,STG IA2,STG IB1,STG IB2,STG IIA,STG IIA1,STG IIA2,STG IIB, STG IIIA,STG IIIB,STG IVA,STG IVB.

\begin{tabular}{|l|l|}
\hline STAGES (STG) & ILLUSTRATION \\
\hline STG I & $\begin{array}{l}\text { Cancer cells start growing and those } \\
\text { tissues will reach the cervix lining. }\end{array}$ \\
\hline STG II & $\begin{array}{l}\text { Cancer cells spreads to the nearby } \\
\text { areas of vagina and cervix and cells } \\
\text { present only in the pelvic region. }\end{array}$ \\
\hline STG III & $\begin{array}{l}\text { Cells spread till pelvic walls causes } \\
\text { swelling in the kidney regions. }\end{array}$ \\
\hline STG IV & $\begin{array}{l}\text { Spreads to bladder and goes till it } \\
\text { reaches the lymph nodes. }\end{array}$ \\
\hline
\end{tabular}

TABLE 1: STAGES OF CANCER CELLS.

In addition to this manual mehods cervical cancers can also be detected using wireless sensor networks and machine learning techniques and also it is used to examine the infections. In ML techniques specifically we use artificial neural networks(ANN) algorithms to predict the cancer rate easily with the data samples collected. In this 
implementation, we enhance algorithms which combines each data samples with the terms. It provide high precise user rate with the accuracy of $90 \%$ [5] accurate results.

Experimental results show that compared with the results of common manual methods, the following are the recent works submitted.

The diagrammatic representation of artificial neural networks is as follows,

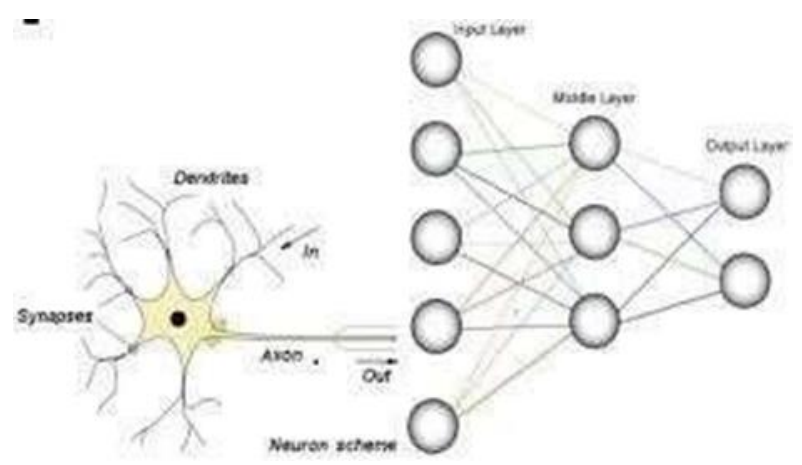

FIG 1:BIOLOGICAL NEURONS.

The objectives of the paper are

1. Decrease the death rate of women dying because of this cervical cancer.

2. Provide accurate results for the infected patients.

3. Analyze the patients reports by the Experts gynecologist in order to identify the severity of the disease.

4. Here we identify the stages of the cancer for proper treatment.

\section{Related Works for Cancer Detection}

The major related works for cancer detection falls on,

SUPPORT VECTOR MACHINE(SVM):

SVM is a supervised machine learning algorithms which is used for classification and regression analysis. This system was proposed by Vapnik along with his colleagues at AT\&T BELL laboratory in 1997.This method is consider as the most reliable method in machine learning.

SVM functions on two categories

o Algorithm allocates fresh samples to the first category.

o Other method is to make non-probabilistic binary classifier.

This system maps the space training examples to point which is used to maximize the width of the both category.

SVM constructs a hyperplane (or) indefinite spaces which is used for

- Classification

- Regression

- Outlier detection.

Simply it is denoted as , $\sum$ ai $\mathrm{k}(\mathrm{xi}, \mathrm{x})=$ constant

Sum of the kernels above can be used to measure the relative nearness of each text.

The diagrammatic representation about support vector machine is as follows,

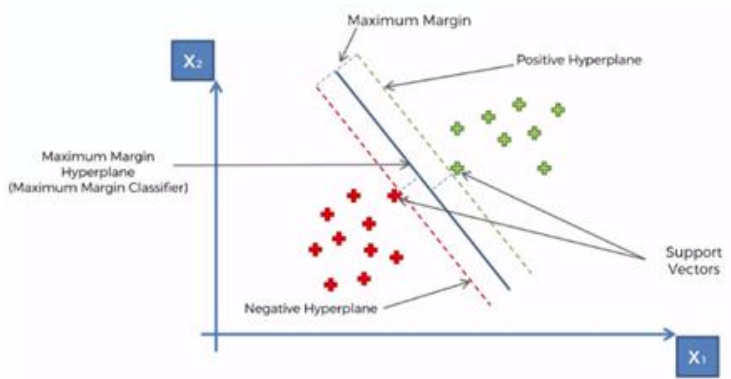

APPLICATIONS:

FIG 2: SUPPORT VECTOR MACHINE.

- This system helps in classifying the text and hypertext.

- This system also helps in classifying the images.

- $\quad$ SVM is used for recognizing the hand written characters.

- This system is also widely applied in biological and other sciences.

- Permutation test based on svm weights have been suggested

TYPES: 
1. Linear SVM

2. Non-linear SVM.

1.LINEAR SVM:

We are given a training dataset of $n$ points,

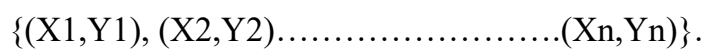

Where Yn are either 1 (or) -1,each indicating the class to which the point X1 belongs .

Each $\mathrm{X}$ term is a $\mathrm{P}$-dimensional real vector.

The two major classification of linear svm includes,

1.Hard margin.

2.Soft margin.

- Hard margin is used for linearly separable data .

- $\quad$ Soft margin is used for non-linearly separable data.

Linear SVM is represented as,

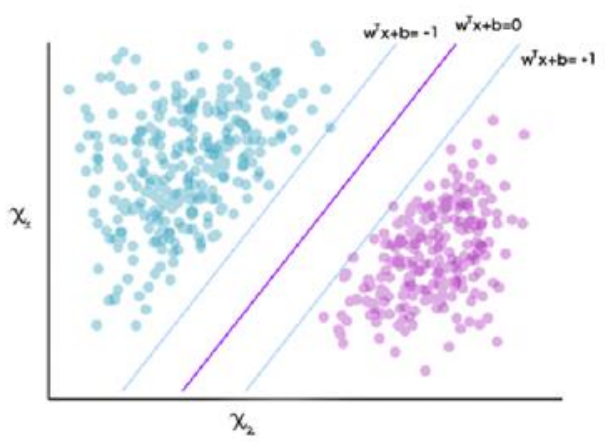

FIG 3: LINEAR SVM.

\section{NON-LINEAR SVM:}

NON Linear svm is classified based on ,

Polynomial(homogenous)

Polynomial(in homogenous)

ADVANTAGES:

- Support Vector Machine functions admirably when there is a reasonable edge of separation between classes.

- It is more profitable in high dimensional spaces.

- It is compelling in instances where the quantity of measurements is bigger than the quantity of examples.

- $\quad$ SVM is equivalent to memory methodical .

DISADVANTAGES:

- Support Vector Machine algorithm does not works adequate for huge informational indexes.

- It does not execute very well when the informational collection has more stable for example target classes. i.e) Overlapping classes.

\section{Proposed Analysis}

Our proposed work for this approach is Artificial Neural Networks (ANN), The detailed description about this approach ia as follows,

Fake Neural Network (ANN) is a profound learning calculation that arose and advanced from the possibility of Biological Neural Networks of human minds. An endeavor to mimic the functions of the human mind finished in the development of ANN. ANN works very much like the organic neural organizations however doesn't by and large take after its activities[8].

A fake neural organization (ANN) is the piece of a figuring framework intended to reenact the manner in which the human cerebrum breaks down and measures data.

It is the establishment of man-made brainpower (AI) and takes care of issues that would demonstrate incomprehensible or troublesome by human or factual principles.

The diagrammatic representation of Artificial neural networks(ANN) is,

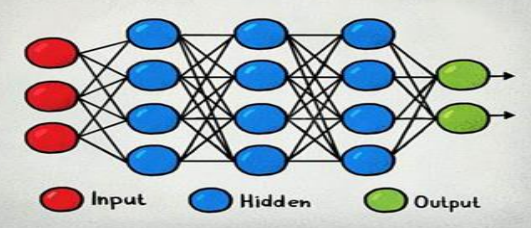

FIG 4: ARTIFICIAL NEURAL NETWORKS. 


\section{TYPES:}

\section{FEEDFORWARD NEURAL NETWORK:}

The feedforward neural organization is quite possibly the most essential counterfeit neural organizations. In this ANN, the information or the information gave ravels a solitary way. It goes into the ANN through the information layer and exits through the yield layer while covered up layers could possibly exist.

\section{RECURRENT NEURAL NETWORKS:}

The Recurrent Neural Network saves the yield of a layer and feeds this yield back to the contribution to all the more likely anticipate the result of the layer. The main layer in the RNN is very like the feed-forward neural organization and the intermittent neural organization begins once the yield of the primary layer is processed.

3.CONVOLUTIONAL NEURAL NETWORKS:

A Convolutional neural organization has a few likenesses to the feed-forward neural organization, where the associations between units have loads that decide the impact of one unit on another unit. In any case, a CNN has at least one than one convolutional layers that utilization a convolution procedure on the information and afterward pass the outcome acquired as yield to the following layer.

4.MODULAR NETWORK:

A Modular Neural Network contains an assortment of various neural organizations that work freely towards getting the yield with no collaboration between them. Every one of the distinctive neural organizations plays out an alternate sub-task by acquiring special information sources contrasted with different organizations.

5.RADIAL BASIS FUNCTION NEURAL NETWORKS:

Radial basis capacities are those capacities that consider the distance of a point concerning the middle. RBF capacities have two layers. In the main layer, the information is planned into all the Radial premise capacities in the secret layer and afterward the yield layer processes the yield in the following stage.

USES:

Artificial Neural Network(ANN) uses the processing of the brain as a basis to develop algorithms that can be used to model complex patterns and prediction problems. ... In our brain, there are billions of cells called neurons, which processes information in the form of electric signals.

ANN algorithm would accept only numeric and structured data as input. To accept unstructured and nonnumeric data formats such as Image, Text, and Speech, Convolutional Neural Networks (CNN), and Recursive Neural Networks (RNN) are used respectively. In this post, we concentrate only on Artificial Neural Networks.

APPLICATIONS OF ARTIFICIAL NEURAL NETWORKS:

Various applications of artificial neural networks(ANN) in medical field:

Health care organizations are leveraging machine-learning techniques, such as artificial neural networks (ANN), to improve delivery of care at a reduced cost. Applications of ANN to diagnosis are well-known; however, ANN are increasingly used to inform health care management decisions. In cancer prediction this ANN plays a major role in classifying the cells. Other than this ANN is involved in,

- $\quad$ Targeted Marketing

- Voice recognition

- Financial

- Intelligent searching

STRUCTURE OF ARTIFICIAL NEURONS AND ITS FUNCTIONS:

- A neural organization with a solitary layer is known as a perceptron. A multi-facet perceptron is called Artificial Neural Networks.

- A Neural organization can have quite a few layers. Each layer can have at least one neurons or units. Every one of the neurons is interconnected with every single other neuron. Each layer could have diverse enactment works also. ANN comprises of two stages Forward spread and Backpropagation. The forward engendering includes duplicating loads, adding predisposition, and applying actuation capacity to the data sources and spreading it forward.

- The backpropagation step is the main advance which typically includes finding ideal boundaries for the model by spreading the retrogressive way of the Neural organization layers. The backpropagation requires improvement capacity to track down the ideal loads for the model.

- ANN can be applied to both Regression and Classification assignments by changing the enactment elements of the yield layers likewise. (Sigmoid enactment work for double characterization, Softmax initiation work for multi-class arrangement and Linear actuation work for Regression).

\section{Experimental Results}

Our experimental results is based on the datasets collected from KAGGLE[30].Our datasets consists of 32 risk factors and 4 target variables which is used to detect the cervical tumors.

After the analysis,

We came to the conclusion that the minimum age of women having the risk of cervical tumors prediction is 26. Here shows the distribution of ages, 


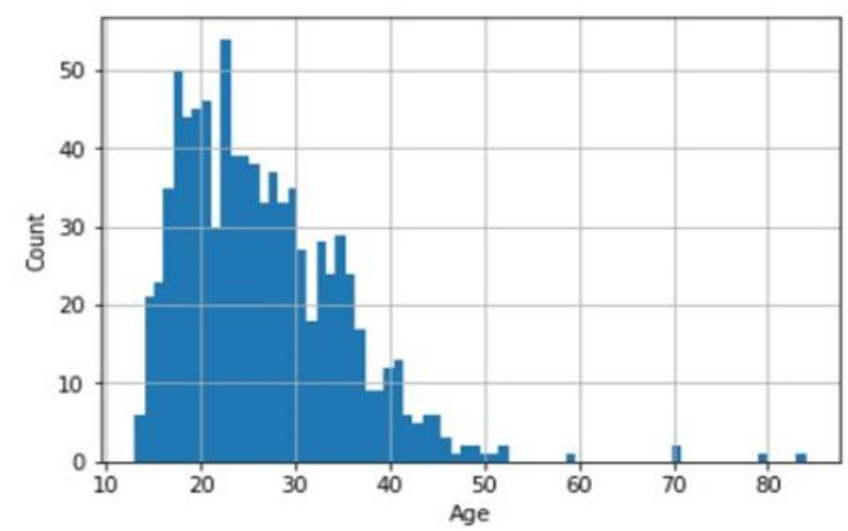

FIG 5:RISK OF CANCER BASED ON AGE.

The above graph shows the results between the age and count.The highest chance of having the cervical cancer ranges from 20 to 35 .

The results of this experiment is based on the target variable, whereas the target variable is collected from the datasets[30].

The expression of cancer prediction is given as,

Cancer prediction=Hinselmann+Schiller+Citology+Biopsy

These target variables gives different values from 0 to 4 .

- 0 represents NO risk factor.

- 4 represents HIGH risk factor.

The final target values ranges between $0,1,2,3,4$.

\begin{tabular}{|l|l|}
\hline CLASS & TARGET COUNT \\
\hline 4 (HIGH RISK) & 6 \\
\hline 3 & 33 \\
\hline 2 & 41 \\
\hline 1 & 28 \\
\hline 0 (NO RISK) & 750 \\
\hline
\end{tabular}

TABLE 2:TARGET VARIABLES.

Since class 4 have less number of variables for testing the data, So here we use Oversampled datas.

Results of oversampled data is as follows,

\begin{tabular}{|l|l|l|l|l|}
\hline & BM & BM & $\begin{array}{l}26 \\
\text { FEATURES }\end{array}$ & $\begin{array}{l}26 \\
\text { FEATURES }\end{array}$ \\
\hline Classifiers & SVM & ANN & SVM & ANN \\
\hline Accuracy & 0.86 & 0.95 & 0.86 & 0.95 \\
\hline Precision & 0.84 & 0.93 & 0.84 & 0.94 \\
\hline F1-Score & 0.85 & 0.94 & 0.86 & 0.95 \\
\hline
\end{tabular}

TABLE 3:OVERSAMPLED DATA.

And the results of the non-oversampled data is as follows,

\begin{tabular}{|l|l|l|l|l|}
\hline & BM & BM & $\begin{array}{l}\text { 26 } \\
\text { FEATURES }\end{array}$ & 26FEATURES \\
\hline Classifiers & SVM & ANN & SVM & ANN \\
\hline Accuracy & 0.89 & 0.88 & 0.89 & 0.88 \\
\hline Precision & 0.76 & 0.7 & 0.76 & 0.79 \\
\hline F1-Score & 0.84 & 0.84 & 0.84 & 0.84 \\
\hline
\end{tabular}

BM-Baseline model .

TABLE 4:NON-OVERSAMPLED DATA.

Here are some of the results of our experiments based on the datasets collected.

\section{Conclusion}

This system provides accurate results of classifying the normal, abnormal and dangerous cells by applying the artificial neural networks(ANN) techniques for the collected data sets. While applying these algorithms this provides higher accuracy when compared to other algorithms[5]. This system is very helpful for the society in identifying the patients with the cervical cancer because this disease is caused without any manifestations. This type of cancer occurs for the women within the age of 30 to 69.This system reduces the risk rate of the patients and the death rate caused due to cervical cancer is also decreased. Several ANN techniques are used for accurate results based on the performance of the algorithm. Our experimental system provides more precise consequences than other manual dissemination methods such as papanicolaou tests and this system is cost effective for the society and it is very helpful for each and every individuals. 


\section{References}

1. R. D. Aldian, E. Purwanti and M. A. Bustomi, Applied Computing Based Artificial Neural Network for Classification of Cervical Cancer, pp. 4-7, (2013).

2. N. Wickramasinghe, L. Al-Hakim, C. Gonzalez and J. Tan, Service S. (Online. (2014). Lean Thinking for Healthcare, XXXII, 645 p. 178 illus.

A. S. Phatak and B, P. A., Classification of Mr Images of Cervical Cancer Using SVM and ANN Engineering, issue 2277, (2015).

3. Comparison of Classification Techniques for Pap Smear Diagnosis. (N.D.), pp. 11-18.

4. N.Mustafa, N.A. M.Isa andM. Y.,Mashor, Campus, (E.n.d.).New Features of Cervical Cells for Cervical Cancer Diagnostic System Using Neural Network, pp. 2-5.

5. B. Sokouti, S. Haghipour and A. D. Tabrizi, A Framework for Diagnosing Cervical Cancer Disease Based on Feedforward MLP Neural Network and ThinPrep Histopathological Cell Image Features, Neural Computing and Applications, vol. 24(1), pp. 221-232, (2014).

6. M. A. Flores, C. Ram'ırez and V. M. Castãno, Image Processing and Neural Networks for Early Detection of Histological Changes, Micai, pp. 632-641, (2004).

7. Y. Jusman and S. Sulaiman, Capability of New Features from FTIR Spectral of Cervical Cells for Cervical Precancerous Diagnostic System Using MLP Networks, Tencon 2009-2009 ..., pp. 1-6, (2009).

8. P. Pouletaut, Ip1, pp. 771-774, (2002). [10] N. Ganesan, Application of Neural Networks in Diagnosing Cancer Disease Using Demographic Data, vol. 1(26), pp. 76-85, (2010).

9. P. J. Lisboa and A. F. G. Taktak, The Use of Artificial Neural Networks in Decision Support in Cancer?: A Systematic Review, vol. 19, pp. 408-415. (2006).

10. P. J. G. Lisboa, A Review of Evidence of Health Benefit from Artificial Neural Networks in Medical Intervention, vol. 15, (2002).

11. C. 12.Mlp, V. Extreme, L. Machine and C. Cell, Classification, I. Cascaded MLP Versus Extreme Learning Machine on , (Isspa), pp. 308-311, (2010).

12. D. A. Ramli, A. F. Kadmin and M. Y. Mashor, Diagnosis of Cervical Cancer Using Hybrid Multilayered Perceptron (HMLP) Network, pp. 591-598, (2004).

13. M. E. G'omez-mayorga and F. J. Gallegos-funes, (n.d.), Diagnosis of Cervical Cancer Using the Median M-Type Radial Basis Function (MMRBF) Neural Network, (Mm), pp.

14. 258-267.

15. W. Xiaoning, Z. Jianwei, X. Yue, W. Wanpeng and L. Minchao, LCT Image Recognition for Cervical Cells Based on BP Neural Network, pp. 1479-1483, (2012).

16. M. Bazoon, D. A. Stacey, C. Cui and G. Harauz, A Hierarchical Artificial Neural Network System for the Classification of Cervical Cells Classification with ANNs, pp. 3525-3529, (1993).

17. M. Kusy, B. Obrzut and J. Kluska, Application of Gene Expression Programming and Neural Networks to Predict Adverse Events of Radical Hysterectomy in Cervical Cancer Patients, Medical \& Biological Engineering \& Computing, vol. 51(12), pp. 1357-65, (2013).

18. K. Tumer, N. Ramanujam, J. Ghosh and R. RichardsKortum, Ensembles of Radial Basis Function Networks for Spectroscopic Detection of Cervical Precancer, IEEE Transactions on Bio-Medical Engineering, vol. 45(8), pp. 953-961, (1998)

19. F. J., Gallegos-Funes, M. E. G'omezMayorga, J. L., Lopez-Bonilla and R. Cruz-Santiago, Rank MType Radial Basis Function (RMRBF) Neural Network for Pap Smear Microscopic Image Classification, Apeiron, vol. 16(4), pp. 542-554, (2009).

20. M. T. а. D. M. Akram Alyass, "From big data analysis to personalized medicine for all: challenges and opportunities," BMC Medical Genomics, vol. 8, no. 33, 2015.

21. S. G. Sunny Sharma, "Decision Tree approach in Machine Learning for Prediction of Cervical Cancer Stages using WEKA," International Journal of Recent Trends in Engineering \& Research, vol. 2, no. 8, 2016.

22. U. Gogate and J. Bakal, "Healthcare Monitoring System Based on Wireless Sensor Network for Cardiac Patients," Biomedical and Pharmacology Journal, vol. 11, no. 3, p. September, 2018.

23. M. Hassanalieragh, A. Page, T. Soyata, G. Sharma, M. Aktas, G. Mateos, B. Kantarci and S. Andreescu, "Health Monitoring and Management Using Internet-ofThings (IoT) Sensing with Cloud-Based Processing: Opportunities and Challenges," in 2015 IEEE International Conference on Services Computing, New York, NY, USA, 2015.

24. Y. J. Fan, Y. H. Yin, L. D. Xu, Y. Zeng and F. Wu, "IoT-Based Smart Rehabilitation System," EEE Transactions on Industrial Informatics, vol. 10, no. 2, pp. 1568-1577, 2014.

25. W. M. O. X. L. Asmaa S. Hussein, "Accurate and Reliable Recommender System for Chronic Disease Diagnosis," in The First International Conference on Global Health Challenges, 2012.

26. InformedHealth.org, "Cervical cancer: Overview," 14 Dec 2012. [Online]. Available: https://www.ncbi.nlm.nih.gov/books/NBK279259/. [Accessed Jan 2019]. 
27. S. Allwin, S. P. K. Kenny and V. Manian, "Classification of stages of maligancies using textron signatures of a cervical cyto image," in 2010 IEEE International Conference on Computational Intelligence and Computing Research, Coimbatore, India, 28-29 Dec. 2010.

28. Rahmadwati, G. Naghdy, M. Ross, C. Todd and E. Norachmawati, "Classification Cervical Cancer Using Histology Images," in 2010 Second International Conference on Computer Engineering and Applications, Bali Island, Indonesia, 12 April 2010.

29. https://www.kaggle.com/loveall/cervical-cancer-risk-classification .

30. P. J. Snijders, R. D. Steenbergen, D. A. Heideman and C. J. Meijer, "HPV-mediated cervical carcinogenesis: concepts and clinical implications, "J Pathol, vol. 208, no. 2, pp. 152-164, Jan, 2016.

31. S. C. Janga and M. M. R. Edupuganti, "Systems and Network-Based Approaches for Personalized Medicine," Current Synthetic and Systems Biology, vol. 2, no. 3, p. 1000e109, 2014.

32. N. K. T. T. N. G. J. G. B. N. P. L. H. T. AmirMohammad Rahmani, "Smart e-Health Gateway: Bringing intelligence to Internet-of-Things based ubiquitous healthcare systems, " in 12th Annual IEEE Consumer Communications and Networking Conference (CCNC), 2015.

A. S. o. C. O. (ASCO), "Cervical Cancer: Stages," Cancer.Net Editorial Board, July 2017. [Online]. Available: https://www.cancer.net/cancertypes/cervical-cancer/stages. [Accessed 01 Jan 2019].

B. Balleyguier, E. Sala, D. C. T, A. Bergman, B. Brkljacic, F. Danza, R. Forstner, B. Hamm, R. KubikHuch, C. Lopez, R. Manfredi, J. McHugo, L. Oleaga, K. Togashi and K. Kinkel, "Staging of uterine cervical cancer with MRI: guidelines of the European Society of Urogenital Radiology," Eur Radiol., vol. 21, no. 5, pp. 1102-1110, 2011.

33. K. Kim, D. H. Song and G. Kim, Nucleus Segmentation and Recognition of Uterine Cervical Pap-Smears Using Region Growing Technique and Fuzzy RBF Network, Image (Rochester, N.Y.), pp. 153-160, (2007).

34. Y. Song, L. Zhang, S. Chen, D. Ni, B. Li, Y. Zhou and T. Wang, A Deep Learning Based Framework for Accurate Segmentation of Cervical Cytoplasm and Nuclei, 36th Annual International Conference of the IEEE Engineering in Medicince and Biology Society, pp. 2903-2906, (2014).

A. N. Bondarenko and A. V. Katsuk, Application of SelfOrganization Maps to the Biomedical Images Classification, IEEE International Siberian Conference on Control and Communications, SIBCON-2007; Proceedings, pp. 131-135, (2007).

35. Z.L.Z.Liand K.Najarian, Automated Classification of PapSmear Tests UsingNeural Networks, IJCNN'01. International Joint Conference on Neural Networks. Proceedings (Cat. No.01CH37222), vol. 4, pp. 2899-2901, (2001).

36. N. Bondarenko, Extracting Feature Vectors of Biomedical Images 'L, pp. 52-56, (2005).

37. J.Hallinan, EvolvingNeuralNetworksfortheClassification ofMalignancy AssociatedChanges, Intelligent DataEngineering andAutomated Learning Ideal 2005, Proceedings, vol. 3578, pp. 382-389, (2005).

38. N. Ampazis, G. Dounias and J. Jantzen, Pap-Smear Classification Using Efficient Second Order Neural Network Training Algorithms, pp. 230-245, (2004).

39. P.Mitra, S. Mitra and S.K. Pal, Staging of Cervical Cancer with Soft Computing, IEEETransactions on Biomedical Engineering, vol. 47(7), pp. 934-940, (2000).

40. P. Mitra, S. Mitra and S. K. Pal, Evolutionary Modular MLP with Rough Sets and ID3 Algorithm for Staging of Cervical Cancer, pp. 67-76,(2001).

41. T. Ochi, K. Murase, T. Fujii, M. Kawamura, J. Ikezoe and T., O.J.,I.,Survival Prediction Using Artificial Neural Networks in Patients with Uterine Cervical Cancer Treated by Radiation Therapy Alone, International Journal of Clinical Oncology, vol. 7(5), pp. 294-300, (2002). 